\title{
Shock-reshock of zirconium to explore the effect of microstructure on the alpha-omega phase transformation
}

\author{
Benjamin M. Morrow ${ }^{1 *}$, Juan P. Escobedo-Diaz ${ }^{2}$, David R. Jones ${ }^{1}$, Carl P. Trujillo ${ }^{1}$, Daniel \\ T. Martinez ${ }^{1}$, Paulo A. Rigg ${ }^{3}$, Cayla R. Harvey ${ }^{1}$, George T. Gray III ${ }^{1}$, and Ellen K. Cerreta ${ }^{1}$ \\ ${ }^{1}$ Los Alamos National Laboratory, Materials Science \& Technology, PO Box 1663 Los Alamos, NM \\ 87545, USA \\ ${ }^{2}$ The University of New South Wales, School of Engineering and Information Technology, Canberra, \\ ACT 2600, Australia \\ ${ }^{3}$ Dynamic Compression Sector, Institute for Shock Physics, Washington State University, Argonne, \\ IL 60439, USA
}

\begin{abstract}
Phase transformations play an important role in the mechanical behavior of materials subjected to extreme loading conditions. A series of shock-reshock experiments were fielded to determine whether the phase transitions in materials are significantly enhanced or inhibited by preexisting microstructural features. Polycrystalline zirconium samples were shock loaded using gas-gun plate impact and soft recovered to examine microstructure using electron backscatter diffraction (EBSD). Drive conditions were varied to study the (hcp) alpha to (hexagonal) omega solidsolid phase transformation. Recovered samples were then subjected to a second shock loading event to determine the change in material behavior as a function of pre-shock microstructure. Crystallography of phase fragments in the final microstructure showed that prior twinning (formed during the shock to a peak stress below the transition threshold) appeared to suppress omega formation/retention after reshock. Conversely, when a material was initially shocked into the omega phase field, retained-omega was not found to have a large impact on subsequent omega formation during reshock. This suggests that nucleation and growth of omega phase are important processes, and the relative activity of nucleation vs. growth processes is modified by a pre-existing substructure. Additionally, orientation relationships reveal a reverse transformation pathway (omega to alpha) dominates the final microstructure, suggesting significant grain growth in the omega phase field is possible even for dynamic timescales.
\end{abstract}

*Corresponding author: morrow@lanl.gov 


\section{Introduction}

Phase transformations play an important role in the mechanical behavior of materials subjected to extreme loading conditions. Both newly-generated material phases and deformation mechanisms such as twinning have a large effect on the material at a microstructural level, which in turn drives observed strength and damage phenomena during shock loading. As such, it is critical to understand the evolution of the microstructure as a result of shock loading to enable phase-aware, kinetically-informed models of dynamic mechanical behavior. Previous studies have shown that shock loading causes major microstructural changes in hexagonal close-packed (hcp) metals, with a resulting change in subsequent mechanical response [1-3]. Specifically, the solid-solid phase transformation from hcp alpha phase to hexagonal omega phase is a large driver for changes in mechanical behavior, as omega phase is known to exhibit higher flow stress than alpha phase [1]. It is known that the shock-induced phase transition occurs above $~ 7 \mathrm{GPa}$ in pure $\mathrm{Zr}$, though the mechanism of the transformation (and the crystallography thereof) and kinetics are poorly understood as a function of drive condition. That is to say that the mechanical response during subsequent deformation relies on the microstructural evolution during and after shock, but this evolution has not been fully quantified. Previous work has examined the orientation relationships between $\alpha$ and $\omega$ phases in $\mathrm{Ti}$ and $\mathrm{Zr}[3,4]$. Orientation relationships and habit planes were noted within a single grain of a polycrystalline specimen, but no comprehensive analysis of the phases as a function of drive condition was performed. Through comparison of $\mathrm{Zr}$ and Ti samples that had been shock-loaded and recovered, the transformation pathway was found to be different between these otherwise similar metals. Ti exhibited a forward $\alpha \rightarrow \omega$ phase transition with no evidence of reversion on unloading, while $\mathrm{Zr}$ was dominated by the reverse $\omega \rightarrow \alpha$ phase transition. The pathway is especially important to consider when assessing phase fractions in the post-shock microstructure. If no phase reversion is expected on unloading, as is the case with $\mathrm{Ti}$, the interpretation of measured phase fractions will be fairly straightforward. If some phase reversion is observed, as is the case with $\mathrm{Zr}$, a more careful analysis is necessary to determine the pathway that the microstructure traversed between ambient, shocked, and unloaded conditions. As an extension of this, the effect of initial microstructure on the mechanism and kinetics of the $\alpha-\omega$ phase transition is poorly understood, but the hypothesis is that the initial microstructure (prior to shock loading) plays an important role in the transformation behavior and kinetics. The present work seeks to study the microstructural evolution of $\mathrm{Zr}$ by shock-loading material using different drive conditions (notably peak stress) and different starting microstructures (via a series of shock-reshock tests). The resulting two-phase microstructures are characterized with respect to plasticity (i.e. twinning) and phase transformations, with special attention to phase fractions, orientation, morphology, and texture to determine the effect of the initial microstructure and test conditions on phase transformation.

\section{Experimental Procedures}

\subsection{Materials}

High-purity $\mathrm{Zr}$ samples were used in this work. Table 1 shows the chemical composition of the material. It is important to note the low oxygen content of the material, as oxygen has been shown to affect the phase transition of $\mathrm{Zr}$ in previous studies [5, 6]. Full details of the processing can be found elsewhere [7]. Briefly, crystal-bar Zr was electron beam melted and cast into a cylinder. The cylinder was upset forged in stages into a plate, which was clockrolled and vacuum annealed at $823 \mathrm{~K}$ for $1 \mathrm{hr}$. This process produces a strongly textured 
microstructure where the c-axis of most grains is within a few degrees of the throughthickness (TT) direction of the plate. The final annealing step produces a fully recrystallized microstructure with equiaxed grains, and an average grain size of $\sim 10-20 \mu \mathrm{m}$ [8]. An inverse pole figure (IPF) map and texture plot of the annealed material is shown in Fig. 1. The material exhibits a strong basal texture, as expected from this type of processing. Subsequent samples were machined such that the loading direction was parallel to the plate normal (and therefore loading occurred normal to the basal plane for most grains).

Table 1. Analysis of chemical composition of $\mathrm{Zr}$ material studied, from[7].

\begin{tabular}{|c|c|c|c|c|c|c|c|c|c|}
\hline Element & $\mathbf{O}$ & $\mathbf{C}$ & $\mathbf{N}$ & $\mathbf{F e}$ & $\mathbf{A l}$ & $\mathbf{V}$ & $\mathbf{H f}$ & $\mathbf{T i}$ & $\mathbf{Z r}$ \\
\hline $\begin{array}{c}\text { Amount } \\
\text { (wt ppm) }\end{array}$ & $<50$ & 22 & $<20$ & $<50$ & $<20$ & $<50$ & 35 & $<25$ & Balance \\
\hline
\end{tabular}
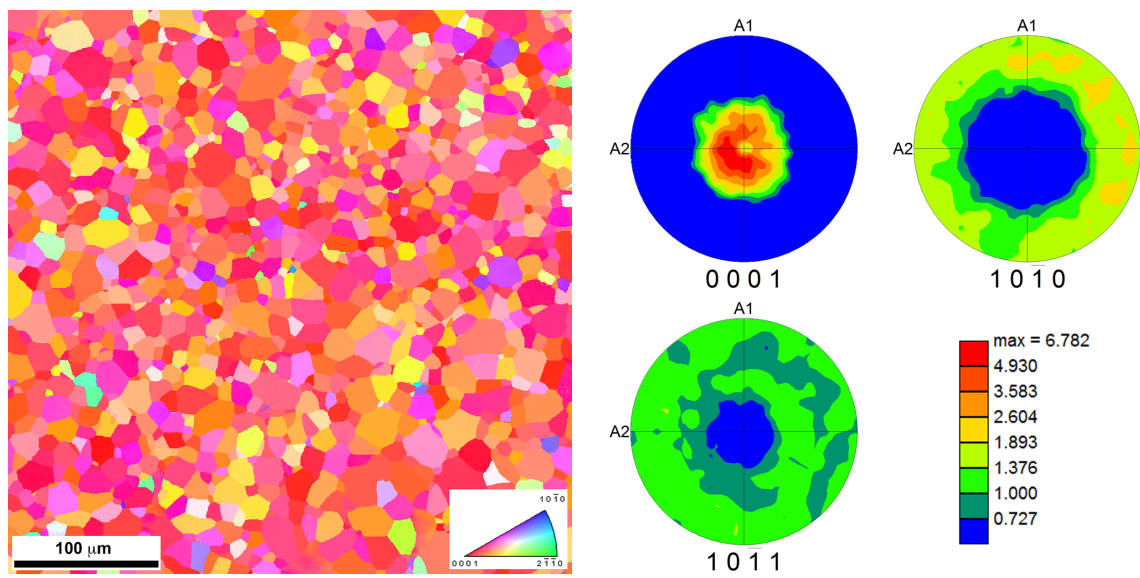

Fig. 1. (a) Inverse pole figure, and (b) texture plot of the annealed $\mathrm{Zr}$ sample used as the initial, unshocked state. Readers are referred to the online version for color.

\subsection{Shock Loading}

To study the effect of microstructure on shock behavior, polycrystalline $\mathrm{Zr}$ samples with varying starting microstructures were shock-loaded via plate impact to a range of peak shock stresses. To generate unique initial microstructures, specimens were shock loaded to different stresses (both above and below the phase transition stress), and soft-recovered, providing material with a different initial microstructure for subsequent shock-loading. Samples were machined into specialized assemblies and shock-loaded using an $80 \mathrm{~mm}$ gas gun equipped with a soft-recovery system [9]. A $\mathrm{Zr}$ flyer plate was accelerated towards the $\mathrm{Zr}$ target assembly at a given velocity, shock-loading the sample on impact. Soft-recovered samples were subjected to post-mortem characterization of the microstructure via EBSD. A limited number of tests were fielded without recovery to simplify velocimetry of the rear surface of the material using photon Doppler velocimetry (PDV) [10] or velocity interferometer system for any reflector (VISAR) $[11,12]$, used to measure material response during dynamic loading. Various features of the phase transformation are more readily apparent using this technique $[6,13]$. The matrix of tests performed in this work is shown in Table 2. 
Table 2. Matrix of shocked and soft-recovered samples of high-purity Zr.

\begin{tabular}{|c|c|c|c|c|}
\hline \multirow{2}{*}{ Specimen } & \multicolumn{2}{|c|}{ Shock } & \multicolumn{2}{c|}{ Reshock } \\
\cline { 2 - 5 } & Peak Stress (GPa) & Velocity (m/s) & Peak Stress (GPa) & Velocity (m/s) \\
\hline A & Unstrained & - & - & - \\
\hline B & 4 & 411.5 & - & - \\
\hline C & 8 & 658 & - & - \\
\hline D & 8.5 & 674 & - & - \\
\hline E & 10 & 825 & - & - \\
\hline F & 10.5 & 834 & - & 658 \\
\hline B2 & 4 & 411.5 & 8 & 825 \\
\hline C2 & 8 & 658 & 10 & \\
\hline
\end{tabular}

\subsection{Microstructural Characterization}

Electron backscatter diffraction (EBSD) was used to measure crystallographic orientations of grains and sub-grain microstructural features before and after shock loading, with emphasis on quantifying the morphology, phase fraction, twinning, and textures. Samples were prepared for EBSD using standard metallographic techniques. Samples were polished with increasingly fine $\mathrm{SiC}$ paper, and final polished with a solution of $45 \mathrm{~mL} \mathrm{H}_{2} \mathrm{O}, 45 \mathrm{~mL}$ $\mathrm{HNO}_{3}$, and $10 \mathrm{~mL} \mathrm{HF}$ applied by aggressively swabbing the surface. Scans were collected using either an FEI XL30, FEI Inspect, or FEI Apreo scanning electron microscope (SEM), each equipped with a EDAX EBSD data collection and analysis system. A baseline of 20 $\mathrm{keV}$ electron beam energy with a $50 \mu \mathrm{m}$ aperture was used, incorporating slight changes for each sample to optimize data collection and scan quality. EBSD was previously used to study plasticity in Zr, especially with regards to twinning [8]. This study also showed that statistics generated by EBSD are a reliable indicator of the overall bulk microstructure.

\section{Results and Discussion}

\subsection{Single Shock Behavior}

Three different stress levels were selected for the initial shock, shown in Table 2, representing low (4 GPa), medium ( $8 \mathrm{GPa})$, and high (10 GPa) stresses. The lowest stress is known to be below the alpha-omega phase transition threshold for shock-loaded $\mathrm{Zr}$ of $7 \mathrm{GPa}$ [1], while the medium and high stresses are above the transition stress. The medium stress samples were slightly above the phase transition pressure and the higher test was well above. It is known that by changing the drive conditions, a different amount of omega phase will form and be retained in the microstructure on unloading, leading to differences in overall microstructure. Such is the case in the present work after a single shock event. Fig. 2 shows IPF maps of the $\mathrm{Zr}$ microstructure in unstrained, single shock, and shock-reshock states. The 4 and $8 \mathrm{GPa}$ shock microstructures were used as the initial microstructure for subsequent shock loading, two of which are shown at the right of Fig. 2. With a single shock below the phase transition (4 GPa, specimen B), the microstructure remains $100 \% \alpha$ phase, with twinning observed as expected. When the material is loaded above the transition stress ( 8 and $10 \mathrm{GPa}$, specimens $\mathrm{C}$ and $\mathrm{E}$, respectively) $\omega$ phase is formed and retained in the microstructure. The amount of omega phase varies based on drive condition, with higher $\omega$ phase fractions observed in the higher stress samples, as reported previously [1]. For these single shock samples above the 

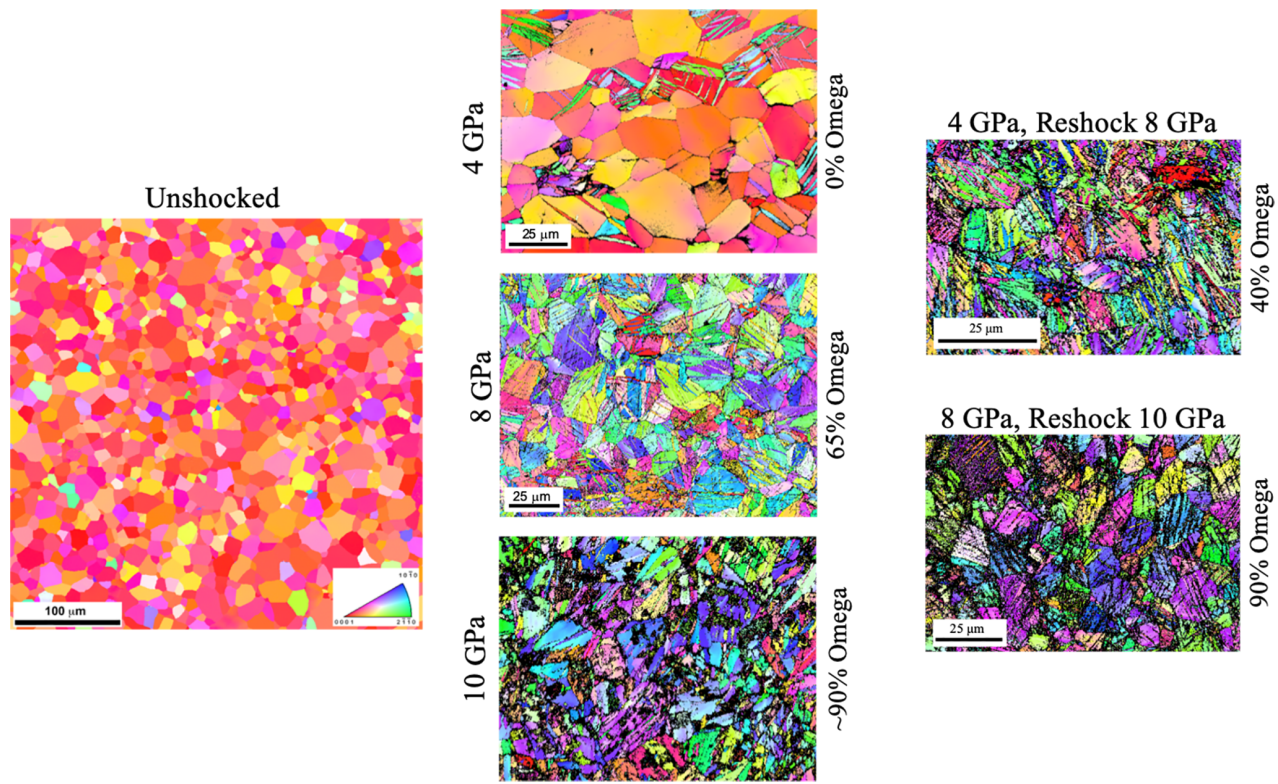

Fig. 2. Inverse pole figure maps of $\mathrm{Zr}$ in unshocked, single shock, and shock-reshock states at conditions specified in Table 2. Readers are referred to the online version for color.

transition, large $\omega$ grains are present, with numerous $\alpha$ lathe variants within, indicating that the grains fully convert to $\omega$ phase then revert some content back to $\alpha$ phase. Generally higher peak stress is associated with a higher amount of residual damage in the microstructure, which appears to frustrate reverse transformation to $\alpha$, as shown in the larger retained $\omega$ phase fraction for higher stresses.

\subsection{Shock-Reshock Behavior}

When the $4 \mathrm{GPa}$ specimen was reloaded to $8 \mathrm{GPa}$ (above the phase transition), $\omega$ phase was retained, but a smaller amount than would be expected from a single shock at $8 \mathrm{GPa}$ alone. As observed with $\mathrm{Ti}$ [14], this may indicate that pre-existing defects can frustrate the nucleation and/or growth of $\omega$ phase. This is supported by the fact that the microstructure of this $\mathrm{Zr}$ sample contained a large number of predominantly $\alpha$ grains (including $\alpha$ twin content) with regions of $\omega$ phase, contrary to observations for a single $8 \mathrm{GPa}$ shock in $\mathrm{Zr}$. Additionally, there are some regions with the characteristic large $\omega$ grains, but the overall mix of phases in the microstructure suggests the microstructure is less well-developed than the samples which were shocked only above the transition stress. When the $8 \mathrm{GPa}$ specimen was reloaded to 10 $\mathrm{GPa}$, the phase fraction of $\omega$ was roughly the same as the $10 \mathrm{GPa}$ single shock microstructure, suggesting that $\omega$ boundaries themselves are weak barriers to subsequent $\omega$ phase formation. If a nucleation and growth process is assumed, most of the nucleation likely occurred during the first shock, leaving only $\omega$ growth processes in the second shock, which likely requires less energy. It is important to note that total $\omega$ formation/retention was not enhanced by the pre-existing $\omega$ content, suggesting that a steady-state microstructure is generated largely as a function of drive condition. This steady state condition appears to be modified by prior microstructure, at least over the duration of a shock loading event, and some features (i.e. $\alpha$ twins/lathes) appear to have a stronger effect than others (i.e. $\omega$ lathes) on $\omega$ phase growth. The active plastic mechanisms (notably dislocation content, twin fraction, and phase fraction) 
likely have different effects on various aspects of the phase nucleation and/or growth processes and, because these are inherently dependent on crystallographic orientations, may be intrinsically linked to the initial microstructural orientation with respect to loading as well.

\section{Conclusions}

High-purity Zr samples with different initial microstructures were subjected to shock loading to determine the effect of prior substructure development on subsequent microstructure formation. Differing levels of twinning and prior phase transformations were present in the samples prior to shock loading. Existing plastic deformation in the $\alpha$ phase (especially twins) was shown to hinder growth of $\omega$ phase, while previously formed omega phase was not observed to be a strong barrier to subsequent $\omega$ growth. Microstructural indicators and velocimetry both suggest that for a given stress condition, the microstructure reaches a steady-state during shock and remains at that state until unloading. Pre-existing $\omega$ in the microstructure did not enhance the amount of retained $\omega$ seen on subsequent shocks, though there is evidence to suggest that higher stresses lead to increased damage in the microstructure which stabilizes the $\omega$ phase and prevents reversion to $\alpha$ on unloading.

Los Alamos National Laboratory is operated by Triad National Security, LLC, for the National Nuclear Security Administration (NNSA) of U.S. Department of Energy (DOE) (Contract 89233218CNA000001). This work was fully funded by Science Campaign 2. LA-UR-21-23570.

\section{References}

1. E.K. Cerreta, J.P. Escobedo, P.A. Rigg, C.P. Trujillo, D.W. Brown, T.A. Sisneros, B. Clausen, M.F. Lopez, T. Lookman, C.A. Bronkhorst, F.L. Addessio, Acta Mater. 61 7712-7719 (2013)

2. D.R. Jones, B.M. Morrow, C.P. Trujillo, G.T. Gray III, E.K. Cerreta, J. Appl. Phys. 122045902 (2017)

3. B.M. Morrow, J.P. Escobedo, R.D. Field, R.M. Dickerson, P.O. Dickerson, C.P. Trujillo, E.K. Cerreta, AIP Conf. Proc. 1793100033 (2017)

4. S.G. Song, G.T. Gray III, Philosophical Magazine, Part A 71 275-290 (1995)

5. E. Cerreta, G.T. Gray III, R.S. Hixson, P.A. Rigg, D.W. Brown, Acta Mater. 53 17511758 (2005)

6. P.A. Rigg, C.W. Greeff, M.D. Knudson, G.T. Gray III, R.S. Hixson, J. Appl. Phys. 106 (2009)

7. G.C. Kaschner, G.T. Gray III, Metall. Mater. Trans. A 31 1997-2003 (2000)

8. B.M. Morrow, R.J. McCabe, E.K. Cerreta, C.N. Tome, Mater. Sci. Eng. A 574 157162 (2013)

9. G.T. Gray III, Shock Wave Testing of Ductile Materials, ASM Handbook Volume 8: Mechanical Testing and Evaluation, ASM International, 2000.

10. O.T. Strand, D.R. Goosman, C. Martinez, T.L. Whitworth, W.W. Kuhlow, Rev. Sci. Instrum. 77 (2006)

11. L.M. Barker, R.E. Hollenbach, J. Appl. Phys. 43 4669-4675 (1972)

12. W.F. Hemsing, Rev. Sci. Instrum. 50 73-78 (1979)

13. B.M. Morrow, P.A. Rigg, D.R. Jones, F.L. Addessio, C.P. Trujillo, R.A. Saavedra, D.T. Martinez, E.K. Cerreta, J. Dyn. Behav. Mater. 3 526-533 (2017)

14. B.M. Morrow, G.T. Gray III, N. Velisavljevic, C.A. Bolme, L.M. Hull, D.R. Jones, T.J. Nizolek, Tech Report: Report on Level 2 Milestone 6522: "Evaluate effect of stress state (DAC, gas gun, HE-drive) on the phase transitions in Ti and Zr", Los Alamos National Laboratory, LA-UR-19-29095 (2019) 